\title{
An Unusual Mid-infrared Flare in a Type 2 AGN: An Obscured Turning-on AGN or Tidal Disruption Event?
}

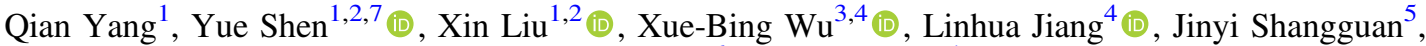 \\ Matthew J. Graham ${ }^{6}$ (1) and $\mathrm{Su} \mathrm{Yao}^{4}$ (1) \\ ${ }^{1}$ Department of Astronomy, University of Illinois at Urbana-Champaign, Urbana, IL 61801, USA; qiany @illinois.edu \\ ${ }^{2}$ National Center for Supercomputing Applications, University of Illinois at Urbana-Champaign, Urbana, IL 61801, USA \\ ${ }^{3}$ Department of Astronomy, School of Physics, Peking University, Beijing 100871, People's Republic of China \\ ${ }^{4}$ Kavli Institute for Astronomy and Astrophysics, Peking University, Beijing 100871, People's Republic of China \\ ${ }_{5}$ Max-Planck-Institut für extraterrestrische Physik, Gießenbachstr. 1, D-85748 Garching, Germany \\ ${ }^{6}$ California Institute of Technology, 1200 E. California Boulevard, Pasadena, CA 91125, USA \\ Received 2019 July 29; revised 2019 September 20; accepted 2019 September 24; published 2019 November 6
}

\begin{abstract}
We report the discovery of an exceptional MIR flare in a Type 2 AGN, SDSS J165726.81+234528.1, at $z=0.059$. This object brightened by 3 mag in the Wide-field Infrared Survey Explorer (WISE) W1 and W2 bands between 2015 and 2017 (and has been fading since 2018), without significant changes $(\lesssim 0.2 \mathrm{mag})$ in the optical over the same period of time. Based on the WISE light curves and near-IR imaging, the flare is more significant at longer wavelengths, suggesting an origin of hot dust emission. The estimated black hole mass $\left(\sim 10^{6.5} M_{\odot}\right)$ from different methods places its peak bolometric luminosity around the Eddington limit. The high luminosity of the MIR flare and its multiyear timescale suggest that it most likely originated from reprocessed dust radiation in an extended torus surrounding the AGN, instead of from stellar explosions. The MIR color variability is consistent with known changing-look AGN and tidal disruption events (TDEs), but inconsistent with normal supernovae. We suggest that it is a turning-on Type 2 AGN or TDE, where the optical variability is obscured by the dust torus during the transition. This MIR flare event reveals a population of dramatic nuclear transients that are missed in the optical.
\end{abstract}

Unified Astronomy Thesaurus concepts: Active galactic nuclei (16); Accretion (14); Tidal disruption (1696)

\section{Introduction}

Modern multiepoch and multiwavelength data have enabled a broad range of time-domain studies from stellar transients to persistent variability from AGN. The multiepoch Wide-field Infrared Survey Explorer (WISE; Wright et al. 2010) data in mid-infrared $W 1 \quad(3.4 \mu \mathrm{m})$ and $W 2(4.6 \mu \mathrm{m})$ bands, in particular, can probe the changes in the continuum emission of warm $(\sim 300 \mathrm{~K})$ or hot $(>800 \mathrm{~K})$ dust in different environments. Mid-infrared (MIR) variability has been seen following optical transient/variability events, such as changing-look AGN (CL AGN; Sheng et al. 2017; Ross et al. 2018; Stern et al. 2018; Yang et al. 2018), tidal disruption events (TDE; Blanchard et al. 2017; Jiang et al. 2017), and supernova (SNe) explosion. In these cases, the MIR emission is predominantly thermal radiation from heated dust grains in the dust torus in AGN or from interstellar medium (ISM)/ circumstellar medium (CSM) in SNe. In rare cases, nonthermal radiation from relativistic electrons accelerated by the shock waves in supernova remnants (Dwek et al. 1987) or by jet launched in radio-loud AGN (Urry \& Padovani 1995) could also make contributions to the observed MIR emission.

The different classes of transients that produce both optical and MIR variability have characteristic spectral features. CL AGN are objects with emerging or disappearing broad emission lines accompanied by large-amplitude continuum variability, possibly caused by changes in the accretion of gas onto the central supermassive black hole (SMBH; LaMassa et al. 2015; MacLeod et al. 2016, 2019; Runnoe et al. 2016; Rumbaugh et al. 2018; Yang et al. 2018). TDEs exhibit emission from helium and/or hydrogen, and some of them additionally show transient iron coronal lines

\footnotetext{
${ }^{7}$ Alfred P. Sloan Research Fellow.
}

when a star is disrupted by the SMBH (Komossa et al. 2008; van Velzen et al. 2011; Gezari et al. 2012; Wang et al. 2012; Arcavi et al. 2014; Holoien et al. 2014, 2016, 2019; Komossa 2015). SNe show strong UV/optical brightening with or without hydrogen, silicon, and helium lines due to the core-collapse of a massive star or the thermonuclear explosion of a white dwarf accreting matter from a companion (e.g., Filippenko 1997; Hillebrandt \& Niemeyer 2000; Heger et al. 2003).

To date, most of these transient events are discovered by their variability from UV/optical or X-ray surveys and classified by follow-up spectroscopic characterization. However, transient events might be hidden within dusty environments and would not be detectable in optical, UV, or soft X-ray. For example, Mattila et al. (2018) reported a dust-enshrouded TDE discovered in the near-IR. The multiepoch imaging from the WISE survey enables systematic discoveries of MIR transient/variable events, which can be cross-correlated with optical light curves. For example, Wang et al. (2018) presented a sample of 14 TDE candidates selecting from AllWISE data with slow declining MIR light curves, while no optical flare is detected in these objects. Assef et al. (2018) identified 45 WISE-selected AGN candidates that are highly variable in the MIR using the AllWISE catalog; only seven of them show significant optical variability.

Here we present an exceptional MIR flare in J165726.81 +234528.1 (hereafter, J1657+2345) from our ongoing systematic study of WISE variability for AGN and galaxies. J1657 +2345 is spectroscopically identified as a Type 2 AGN at $z=0.059$ in SDSS (York et al. 2000). Its MIR light curves remained quiescent until the end of 2015, followed by an exceptionally large flare in both $W 1$ and $W 2$. Among $>1.8$ million spectroscopic galaxies in the SDSS fourteenth data release (DR14, Abolfathi et al. 2018), J1657+2345 is identified 


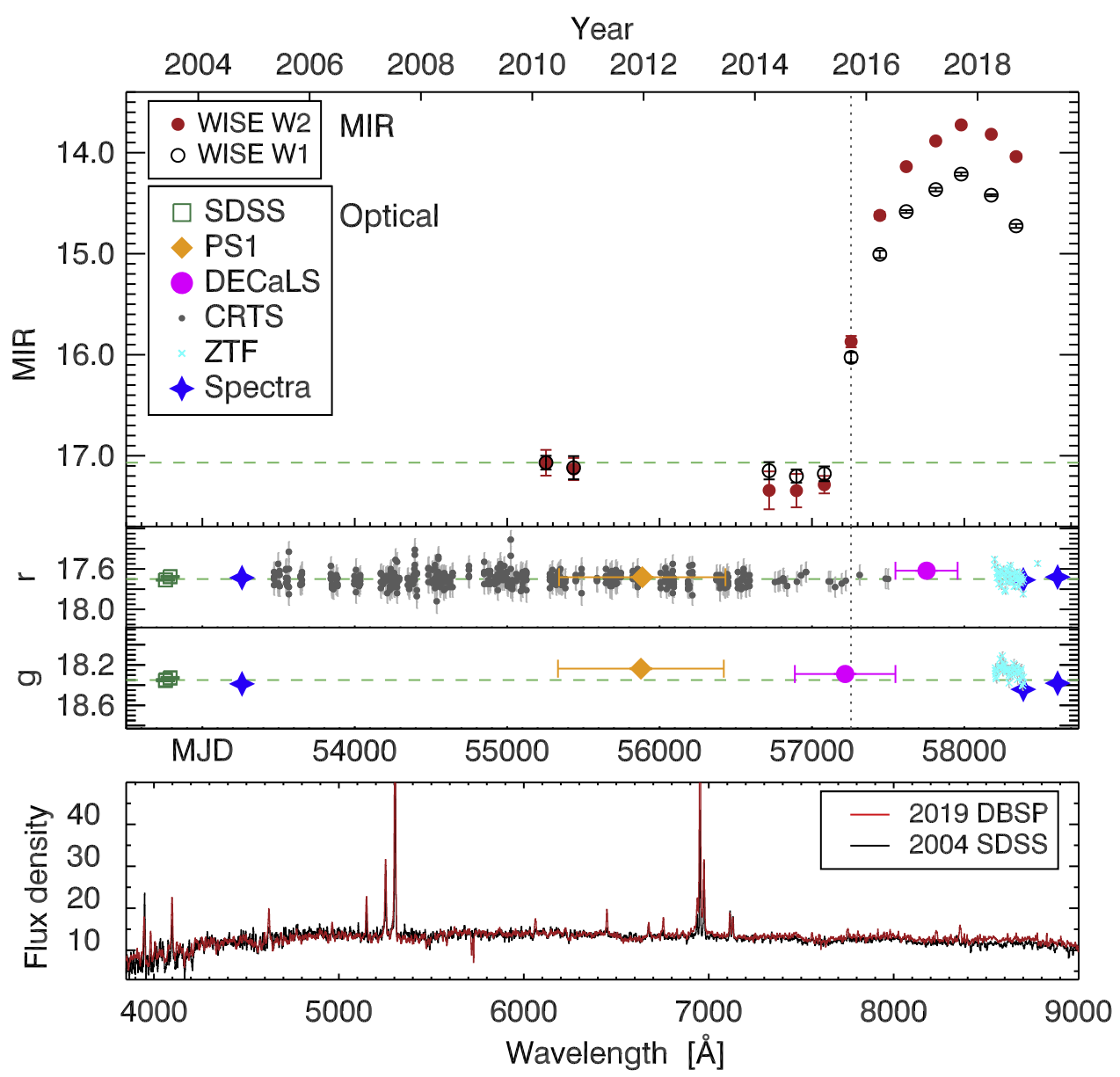

Figure 1. Top panel: light curves of J1657+2345 in MIR from WISE and in optical from various surveys, including SDSS, PS1, DECaLS, CRTS, and ZTF. All magnitudes are $\mathrm{AB}$ magnitudes. To compare the optical and MIR data, the $y$-axes in the three panels are plotted on the same scale. The horizontal dashed lines show the earliest epoch magnitude in $g, r$, and $W 1$ bands from bottom to top panels. The vertical dotted line shows the first WISE epoch when the object began to brighten. $\mathrm{J} 1657+2345$ flared for more than $3 \mathrm{mag}$ in MIR from 2015 to 2017. However, there is no significant variability in the optical from 2003 to 2019 (constant within $0.2 \mathrm{mag}$ ). Bottom panel: Spectra of J1657+2345 taken in 2019 by P200/DBSP (red) and taken in 2004 by SDSS (black). There is no obvious variability between the two optical spectra.

as the most dramatic case with its WISE magnitudes brightened by 2.9 and $3.3 \mathrm{mag}$ (a factor of 13.8 and 21.5 increase in flux) in $W 1$ and $W 2$ bands, respectively, between 2015 and 2017. According to the latest WISE data taken in 2018 August, J1657 +2345 has passed its peak MIR luminosity and is fading (see Figure 1).

We compiled all available optical photometric data from various surveys, including the SDSS, Pan-STARRS (PS1; Chambers et al. 2016), the Dark Energy Camera Legacy Survey (DECaLS; Dey et al. 2019), the Catalina Real-time Transient Survey (CRTS; Drake et al. 2009), and the Zwicky Transient Facility (ZTF; Bellm et al. 2019). The CRTS data 8 months after the onset of the MIR flare shows that it was still quiescent in the optical. To identify potential spectral variability, we obtained new optical spectra in 2018 September and 2019 May. There is no significant difference between the new spectra and the earlier SDSS spectrum taken in 2004 before the MIR flare. The optical photometric data constrain a maximum variability of $\sim 0.2 \mathrm{mag}$ before and after the onset of the MIR flare. Thus this object is markedly different from any previous transients first identified in the optical. It is reminiscent of the handful of MIR variable AGN candidates with low optical variability reported in Assef et al. (2018), but the contrast of the MIR and optical variability is much more extreme. The large difference between the MIR and optical variability motivates a thorough investigation of this event to explore possible scenarios on the nature of the extreme MIRonly flare.

In Section 2, we describe the observations of J1657+2345 in MIR, optical, and near-infrared. We describe the variability, spectral energy distribution (SED), and spectral features of $\mathrm{J} 1657+2345$ in Section 3. In Section 4, we compare its MIR variability to CL AGN, TDEs, and SNe. We discuss the timescales of MIR variability from reprocessing the optical/ UV variability with simple geometric dust torus models. We conclude in Section 5. In this paper, we use a $\Lambda$ CDM cosmology with parameters $\Omega_{\Lambda}=0.7, \Omega_{\mathrm{m}}=0.3$, and $H_{0}=$ $70 \mathrm{~km} \mathrm{~s}^{-1} \mathrm{Mpc}^{-1}$. Unless otherwise specified, all magnitudes are in the AB system (Oke \& Gunn 1983).

\section{Observations and Data}

\subsection{WISE Photometry}

WISE scanned the full sky from 2010 January to July in four bands centered at wavelengths of 3.4, 4.6, 12, and $22 \mu \mathrm{m}$ $(W 1, W 2, W 3$, and $W 4)$. The secondary cryogen survey and 
Table 1

WISE MIR Photometry

\begin{tabular}{|c|c|c|c|c|c|c|}
\hline Date & MJD & Number & $W 1$ & $W 2$ & $W 3$ & $W 4$ \\
\hline 2010 Feb 28 & 55255 & 15 & $17.07(0.07)$ & $17.07(0.13)$ & $15.36(0.16)$ & $14.00(0.28)$ \\
\hline 2010 Aug 28 & 55436 & 17 & $17.12(0.11)$ & $17.13(0.11)$ & $\cdots$ & $\cdots$ \\
\hline 2014 Mar 3 & 56719 & 14 & $17.15(0.09)$ & $17.34(0.19)$ & $\cdots$ & $\cdots$ \\
\hline 2015 Feb 28 & 57081 & 14 & $17.18(0.07)$ & $17.29(0.09)$ & $\cdots$ & $\cdots$ \\
\hline 2015 Aug 23 & 57257 & 17 & $16.03(0.05)$ & $15.87(0.06)$ & $\cdots$ & $\ldots$ \\
\hline 2016 Feb 27 & 57445 & 15 & $15.01(0.04)$ & $14.62(0.03)$ & $\ldots$ & $\ldots$ \\
\hline 2017 Aug 14 & 57979 & 9 & $14.21(0.02)$ & $13.73(0.03)$ & $\ldots$ & $\ldots$ \\
\hline 2018 Feb 28 & 58177 & 15 & $14.42(0.01)$ & $13.82(0.02)$ & $\cdots$ & $\cdots$ \\
\hline 2018 Aug 10 & 58340 & 9 & $14.72(0.02)$ & $14.04(0.03)$ & $\cdots$ & $\cdots$ \\
\hline
\end{tabular}

Note. The WISE magnitudes are converted to $\mathrm{AB}$ magnitudes. The values in the parentheses are the magnitude errors.

Table 2

Optical Photometry

\begin{tabular}{|c|c|c|c|c|c|c|}
\hline Survey & Photometry & MJD & Year & $g$ & $r$ & $z$ \\
\hline SDSS & Model & 52788 & 2003 & $18.33(0.01)$ & $17.68(0.01)$ & $17.05(0.02)$ \\
\hline \multirow[t]{3}{*}{ PS1 } & Kron & $55333-56422$ & 2010-2013 & $18.24(0.01)$ & $\cdots$ & $\cdots$ \\
\hline & & $55341-56433$ & 2010-2013 & $\ldots$ & $17.68(0.01)$ & $\ldots$ \\
\hline & & $55275-56520$ & 2010-2013 & $\cdots$ & $\cdots$ & $17.06(0.01)$ \\
\hline \multirow[t]{3}{*}{ DECaLS } & Model & $56888-57548$ & 2014-2016 & $18.29(0.01)$ & .. & $\cdots$ \\
\hline & & $57548-57956$ & 2016-2017 & $\ldots$ & $17.62(0.01)$ & $\ldots$ \\
\hline & & $57110-57120$ & 2015 & $\cdots$ & $\cdots$ & $17.02(0.01)$ \\
\hline \multirow[t]{2}{*}{ ZTF } & Aperture & $58204-58389$ & 2018 & $18.26(0.04)$ & $\cdots$ & $\cdots$ \\
\hline & & $58198-58482$ & 2018 & $\cdots$ & $17.68(0.03)$ & $\cdots$ \\
\hline CRTS & Aperture(unfiltered) & $53474-57500$ & 2005-2016 & $\cdots$ & $17.68(0.10)$ & $\cdots$ \\
\hline SDSS/spec & Spectrophotometry & 53260 & 2004 & $18.39(0.01)$ & $17.69(0.01)$ & $17.12(0.01)$ \\
\hline $2.16 \mathrm{~m}$ & Spectrophotometry & 58387 & 2018 & $18.44(0.01)$ & $17.71(0.01)$ & $17.26(0.06)$ \\
\hline DBSP & Spectrophotometry & 58612 & 2019 & $18.38(0.01)$ & $17.68(0.01)$ & $17.02(0.01)$ \\
\hline
\end{tabular}

Note. The photometry of PS1 and DECaLS is from stacked images. The magnitudes of ZTF and CRTS in this table are median magnitudes. The compiled light curves show that there is no optical variability (more than $0.2 \mathrm{mag}$ ) from 2003 to 2019.

Near-Earth Object Wide-field Infrared Survey Explorer (NEOWISE; Mainzer et al. 2011) Post-Cryogenic Mission mapped the sky from 2010 August to 2011 February. The NEOWISE Reactivation Mission (NEOWISE-R; Mainzer et al. 2014) surveys the sky in $W 1$ and $W 2$ bands from 2013 twice a year. WISE obtains $\sim 10-20$ observations within a $36 \mathrm{hr}$ window in each visit. We calculate the median magnitude and magnitude error, specifically the semiamplitude of the range enclosing the 16 th and 84th percentiles of all flux measurements within a 6-month window (summarized in Table 1). We limit to good quality single-epoch data points with the best frame image quality score $\left(q i \_f a c t=1\right)$, observed far away from the South Atlantic Anomaly (saa_sep $\geqslant 5$ ), with no contamination from the moon (moon_masked $=0$ ), and excluding spurious detection $(c c$ flags $=0)$. The WISE magnitudes are converted from Vega to $\mathrm{AB}$ magnitude as $m_{\mathrm{AB}}=m_{\mathrm{Vega}}+\Delta m$, where $\Delta m$ is $2.699,3.339,5.174$, and 6.620 in $W 1, W 2, W 3$, and $W 4$ bands, respectively.

\subsection{Optical Photometry}

We compile all available optical photometric data from various surveys, including SDSS, PS1, DECaLS, CRTS, and ZTF (see Table 2). J1657+2345 was observed in the SDSS imaging survey in ugrizsDSs bands in 2003 May. As J1657 +2345 is an extended source at $z=0.059$, we use the SDSS model mag, obtained by fitting to de Vaucouleurs (elliptical galaxies) or exponential (spiral galaxies) models to the photometric data. The SDSS gri magnitudes are nearly AB. The SDSS $u$-band and $z$-band magnitudes are corrected to the $\mathrm{AB}$ system using $u_{\mathrm{AB}}=u_{\mathrm{SDSS}}-0.04$ mag and $z_{\mathrm{AB}}=z_{\mathrm{SDSS}}+$ 0.02 mag (Fukugita et al. 1996). J1657+2345 was observed by PS1 (Chambers et al. 2016) from 2010 March to 2014 June in grizy $_{\mathrm{PS} 1}$ bands. We use the PS1 Kron (1980) magnitude from the PS1 stack catalog. J1657+2345 was observed three times in each band by DECaLS from 2014 August to 2016 June in $g_{\text {DECAM }}$ band (3 epochs), from 2016 June to 2017 July in 
$r_{\text {DECAM }}$ band (3 epochs), and from 2015 March to April in $z_{\text {DECAM }}$ band (3 epochs). These images were stacked and presented in the DECaLS DR7 catalog (Dey et al. 2019). We use the DECaLS model magnitude, obtained by fitting five morphological types including point sources, round exponential galaxies with a variable radius, de Vaucouleurs profiles, exponential profiles, or composite profiles. The CRTS (Drake et al. 2009) repeatedly observed a large portion of the sky. $\mathrm{J} 1657+2345$ was observed more than 450 times by CRTS from 2005 to 2016. The CRTS data are aperture-based, unfiltered photometry. The ZTF (Bellm et al. 2019) is a new time-domain survey from 2017 in gri $_{\mathrm{ZTF}}$ bands. J1657+2345 was covered by ZTF about 50 times in $g$ and $r$ bands from 2018 March to December. The ZTF data are aperture-based photometry with a typical aperture diameter of $2^{\prime \prime}$.

\subsection{Optical Photometric Data Calibration}

To calibrate the optical data from different surveys onto the same flux scale, we apply additive corrections to the optical magnitudes taking into account different filter curves and photometry methods. We convert all magnitudes to the $\mathrm{AB}$ system. To correct for different filter curves, we convolve the DBSP spectrum (see Section 2.4) with the PS1/DECaLS/ZTF filter curves to obtain synthetic magnitudes, and compare to those derived with the SDSS filters to derive the corrections. Thus the calibration offsets for PS1 are $0.09,0.02$, and -0.06 mag in $g r z$ bands; for DECaLS are 0.07, 0.13, and 0.04 mag in grz bands; and for ZTF are 0.06 and 0.13 mag in $g r$ bands. We apply an additional correction for ZTF magnitudes as ZTF uses aperture-based photometry. According to the DECaLS photometry, the offsets between model magnitude and $2^{\prime \prime}$ aperture magnitude for $\mathrm{J} 1657+2345$ are -0.50 and -0.46 mag in $g$ and $r$ bands, respectively. Therefore, ZTF magnitudes are further corrected by -0.44 and $-0.33 \mathrm{mag}$ in $g$ and $r$ bands. CRTS data are aperture-based photometry and observed unfiltered, so we apply a constant offset +0.18 to the CRTS magnitudes to match the median CRTS magnitude (18.50 mag) to the contemporary calibrated PS1 $r$-band magnitude (18.68 mag). We summarize the calibrated grz photometry in Table 2.

\subsection{Spectroscopic Observations}

J1657+2345 was observed by SDSS on 2004 September 12 . The SDSS spectroscopy covers a wavelength range from 3820 to $9185 \AA$ with a spectral resolution of $R=\lambda / \Delta \lambda \sim 2000$ (Abazajian et al. 2009) and a spectral binning of $69 \mathrm{~km} \mathrm{~s}^{-1}$ per pixel. The median signal-to-noise ratio $(\mathrm{S} / \mathrm{N})$ per pixel of the SDSS spectrum for $\mathrm{J} 1657+2345$ is 15 . $\mathrm{J} 1657+2345$ has no obvious broad emission lines in the spectrum. The narrow emission line flux ratios suggest it is photoionized by $\mathrm{AGN}$, with $\log ([\mathrm{O} \mathrm{III} / H \beta)=0.65 \pm 0.01$ and $\log ([\mathrm{N} \mathrm{II}] / H \alpha)=$ $-0.24 \pm 0.01$ (Kauffmann et al. 2003). Therefore, J1657+2345 is a Type 2 AGN, specifically, a Seyfert 2 galaxy according to the division line between Seyferts and LINERs (Cid Fernandes et al. 2010). The stellar velocity dispersion $\sigma_{*}$ is $60.8 \pm 11.2 \mathrm{~km} \mathrm{~s}^{-1}$, measured from the SDSS spectrum (Thomas et al. 2013). Using the local relation between SMBH mass $M_{\mathrm{BH}}$ and $\sigma_{*}$ (Kormendy $\&$ Ho 2013), we estimate a BH mass of $10^{6.2 \pm 0.2} M_{\odot}$. The measured $\sigma_{*}$ may be unreliable given SDSS's spectral resolution, therefore we will use different approaches to cross-check the $\mathrm{BH}$ mass estimate (Section 3).
We obtained an optical spectrum using the Xinglong $2.16 \mathrm{~m}$ telescope in China on 2018 September 26. We use the Beijing Faint Object Spectrograph and Camera (BFOSC) with Grism 4. The object was observed under seeing $\sim 2^{\prime \prime}$, so we used a slit width of 2 ". 3 . This instrument configuration yields a dispersion of $198 \AA \mathrm{mm}^{-1}$, a wavelength coverage from 3850 to $8860 \AA$, and a resolution of $R \sim 265$ (Fan et al. 2016). The object was observed with one exposure of $1800 \mathrm{~s}$. We observed a standard star with the same configuration, HD 161817, for flux calibration. The spectrum was reduced using standard IRAF routines (Tody 1986, 1993). The median $\mathrm{S} / \mathrm{N}$ per pixel of the $2.16 \mathrm{~m}$ spectrum is 6 .

We obtained another optical spectrum using the Palomar P200/DBSP spectrograph on 2019 May 9. We used DBSP with the G600 grating on the blue side with a central wavelength of $4000 \AA$ and G316 grating on the red side with a central wavelength of $7500 \AA$. The object was observed with a 1 !'5 slit under seeing $\sim 1$ !' 2 . This configuration yields a dispersion of $71 \AA \mathrm{mm}^{-1}$ and a resolution of $R \sim 969$ at $4000 \AA$ on the blue side; a dispersion of $135 \AA \mathrm{mm}^{-1}$ and a resolution of $R \sim 958$ at $7500 \AA$ on the red side. We obtained one exposure of $900 \mathrm{~s}$. We observed a standard star, BD $+28 \mathrm{~d} 4211$, for flux calibration. The DBSP spectrum covers a wide wavelength range, and the median $\mathrm{S} / \mathrm{N}$ per pixel from 3800 to $9200 \AA$ is 9 .

The spectra were taken in smaller aperture/slit than photometry, and $\mathrm{J} 1657+2345$ is an extended source. To correct for aperture loss, we calculate spectrophotometry by convolving the spectra with SDSS grz filter curves and compare the spectrophotometry with photometry. We applied a constant scaling factor to the SDSS spectrum, specifically a factor of 1.95 , to match the SDSS spectrophotometry in $r$ band to SDSS $r$-band model photometry. We apply the same scaling factor to other spectra as their [O III] fluxes are consistent with that of the SDSS spectrum (see Section 3.3).

\subsection{Near-infrared Photometry}

$\mathrm{J} 1657+2345$ was observed by the Two Micron All Sky Survey (2MASS; Skrutskie et al. 2006) on 2002 February 12 in $J, H$, and $K s$ bands. We downloaded the $J, H$, and $K_{s}$ band images from NASA/IPAC Infrared Science Archive (IRSA), ${ }^{8}$ and performed aperture photometry on the images using the Python package photutils. ${ }^{9}$ The source-free background is fitted and subtracted with a two-dimensional third-order polynomial function, which is flexible to fit the large-scale background gradient but robust not to fit the small-scale variation due to the source. The flux of the source is integrated over a circular aperture of $4^{\prime \prime}$ in radius, and the local background is measured and removed with an annulus of radii between $25^{\prime \prime}$ and $35^{\prime \prime}$. By increasing the aperture radius until $12^{\prime \prime}$, we find that the $4^{\prime \prime}$ aperture size is large enough to enclose (more than $95 \%$ of the total flux). No aperture correction is applied. Our new measurements are systematically brighter than the 2MASS point-source catalog results, by $0.29,0.32$, and 0.45 magnitude, respectively, for $J, H$, and $K_{s}$ bands. However, our results are more consistent with the optical spectra and match the stellar emission model better (see Section 3.2). We converted the 2MASS Vega magnitudes to $\mathrm{AB}$ magnitude as $m_{\mathrm{AB}}=m_{\mathrm{Vega}}+\Delta m$, where $\Delta m$ is $0.89,1.37$,

\footnotetext{
8 irsa.ipac.caltech.edu/

9 http://photutils.readthedocs.io/
} 
Table 3

Near-infrared Photometry

\begin{tabular}{lcccccc}
\hline \hline Instrument & Date & MJD & $Y$ & $J$ & $H$ \\
\hline 2MASS & 2002 Feb 12 & 52317 & $\ldots$ & $16.85(0.10)$ & $16.68(0.17)$ & $16.40(0.13)$ \\
UKIRT & 2019 Feb 26 & 58540 & $16.99(0.02)$ & $16.72(0.01)$ & $16.40(0.01)$ & $16.02(0.01)$ \\
\hline
\end{tabular}

Note. All magnitudes are converted to AB.

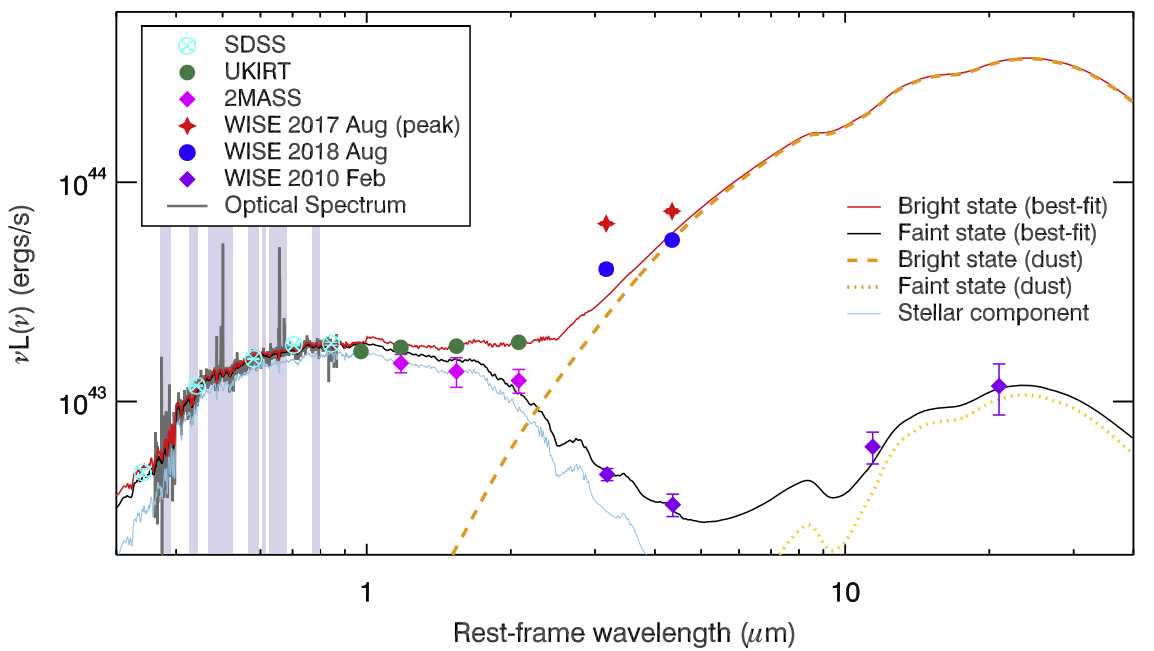

Figure 2. SED model of J1657+2345 in the faint state (black) and the bright state (red). We simultaneously fit the spectral and photometric data. The dark gray line is the SDSS spectrum, rescaled to match the SDSS model magnitude (cyan circles with X). The wavelength ranges with strong emission lines are excluded in the fit (shaded regions). We consider stellar emission (light blue solid line), a power-law continuum (negligible), and a clumpy dust radiative transfer model (orange dashed line in bright state, yellow dotted line in faint state). The red stars are WISE data in 2017 August, which is the brightest WISE epoch. J1657+2345 varies more at redder wavelengths. The hot dust enhanced significantly, while the optical light is not variable.

and 1.84 in $J, H$, and $K_{s}$ bands. We summarize the near-infrared photometry of $\mathrm{J} 1657+2345$ in Table 3.

We obtained new near-infrared imaging using the United Kingdom Infrared Telescope (UKIRT) on 2019 February 26 in YJHK bands. We used the UKIRT Wide Field Camera (WFCAM) with a four-point dithering pattern in $Y$ band, and eight-point dithering pattern in $J H K$ bands. At each pointing the exposure time was $10 \mathrm{~s}$. WFCAM data were processed by the Cambridge Astronomical Survey Unit. We use UKIRT photometry derived from a $4^{\prime \prime}$ radius aperture for comparison with the 2MASS photometry. We converted the UKIRT Vega magnitudes to AB magnitude as $m_{\mathrm{AB}}=m_{\text {Vega }}+\Delta m$, where $\Delta m$ is $0.634,0.938$, 1.379, and 1.900 in $Y J H K$ bands (Hewett et al. 2006).

\section{Results}

\subsection{Variability of $J 1657+2345$}

Figure 1 displays the multiwavelength light curves for J1657 +2345 from our collected data. From 2010 to 2015, J1657+2345 was scanned by WISE five times, during which it remained in the faint state. 2015 August 23 was the first epoch when WISE captured the flare. It flared by 1.15 and $1.42 \mathrm{mag}$ in $W 1$ and $W 2$ bands within half a year from 2015 February to August. It continued to brighten from 2015 to 2017. The peak-luminosity epoch caught by WISE is 2017 August 14, with $W 1=14.21$ and $W 2=13.73$ (AB magnitude). It brightened by 2.97 and 3.56 (a factor of 13.8 and 21.5 flux increase) in $W 1$ and $W 2$ bands during $2.5 \mathrm{yr}$. The WISE observation on 2018 February 28 indicates that it started to fade. It was still in the bright state at the latest public WISE epoch (2018 August 10), 2.46 and 3.25 mag brighter than its faint state, in $W 1$ and $W 2$ bands.
However, there is no significant variability in the optical from 2003 to 2019. The optical photometric data from various surveys (summarized in Table 2) are consistent with each other within $\sim 0.2 \mathrm{mag}$. The continuous CRTS data from 2005 to 2016 is constant with a standard deviation of $0.07 \mathrm{mag}$. The stacked DECaLS $r$-band photometry from 2016 June 9 to 2017 July 22 is consistent with the SDSS and PS1 photometry within $0.1 \mathrm{mag}$. The ZTF data from 2018 March to December is also consistent with the SDSS, PS1, and DECaLS photometry within 0.1 mag. Furthermore, we find no evidence for significant flux variations between the SDSS spectrum, the $2.16 \mathrm{~m}$ telescope spectrum, and the DBSP spectrum that were taken $\sim 15 \mathrm{yr}$ apart. There is no continuum flux enhancement in the optical spectra compared to its earlier SDSS spectrum (we discuss the details on spectra in Section 3.3).

\subsection{SED Fitting}

$\mathrm{J} 1657+2345$ brightened by $0.6 \mathrm{mag}$ more in the redder $W 2$ band than in $W 1$ band, a factor of 1.6 more in flux. It brightened by $0.13,0.28$, and $0.38 \mathrm{mag}$ in $J, H$, and $K$ bands, respectively, comparing the 2MASS and UKIRT photometry. Therefore we confirmed that the J1657+2345 flare is more prominent at longer IR wavelengths.

We construct SEDs well before (faint state) and after (bright state) the onset of the flare. We simultaneously fit the optical spectrum and IR photometric data for each state. In the faint state, we use the SDSS spectrum (2004 September), 2MASS photometry (2002 February), and WISE data (2010 February). In the bright state, we use the DBSP spectrum (2019 May), UKIRT photometry (2019 February), and the latest WISE data (2018 August). 
Figure 2 shows the SED fitting results in both states. To match the IR photometry with larger apertures, the SDSS spectrum (dark gray line) is scaled by a factor of 1.95 to match the SDSS model mag (as described in Section 2.4). We only use segments of continuum without strong line emission. ${ }^{10}$ We perform the SED fitting with a Markov chain Monte Carlo (MCMC) method (Shangguan et al. 2018). We adopt the host galaxy stellar emission model that consists of two simple stellar population models (Bruzual \& Charlot 2003) with the Chabrier (2003) initial mass function. The young stellar population has an age less than $300 \mathrm{Myr}$ and the old stellar population has an age of 0.3-15 Gyr. The stellar masses and ages are free parameters in the fitting. We incorporate a power-law component to fit the scattered UV emission from the accretion disk, which is found necessary for Type 2 AGN (Bessiere et al. 2017; Zhao et al. 2019). The amplitude and the slope of the power-law model are free parameters in the fitting. A clumpy dust torus model (Hönig \& Kishimoto 2017) is used to mainly fit the MIR data. The model templates are calculated with a Monte Carlo radiative transfer method. Hönig \& Kishimoto (2017) provided model templates both with and without a wind component. Since we do not have sufficient MIR coverage to fit the torus component, we chose to use the simpler model without the wind to reduce the number of free parameters. Shangguan \& Ho (2019) found that this model is able to reproduce the IR SED of type 2 AGNs. The fitting parameters are the inclination angle, the power-law index of the cloud radial distribution, the number of clouds on the equatorial plane, and the vertical scale height. The degeneracy in the geometric parameters makes it difficult to provide a unique physical interpretation of the best-fit torus model (Zhuang et al. 2018), especially with our limited data coverage. Therefore we only use the successful model fits to estimate the torus luminosity.

The results show that the host galaxy is dominated by the old stellar population, with stellar mass $M_{* \text {,old }}=10^{9.59 \pm 0.02} M_{\odot}$ and age $1.45 \pm 0.04 \mathrm{Gyr}$ (summarized in Table 4). Using the relation between stellar mass and black hole mass of AGN in the nearby universe (Reines \& Volonteri 2015), we estimate a black hole mass of $10^{5.97 \pm 0.30} M_{\odot}$, consistent with our earlier estimation using the $M-\sigma_{*}$ relation.

Since $\mathrm{J} 1657+2345$ is highly obscured in the optical, we use its MIR flux to estimate the bolometric luminosity, $L_{\mathrm{bol}}$, at different epochs. We first estimate the $6 \mu \mathrm{m}$ luminosity, $\lambda L_{\lambda}(6 \mu \mathrm{m})$, based on our SED fit. We then adopt $L(2-10 \mathrm{keV}) /$ $\lambda L_{\lambda}(6 \mu \mathrm{m})=0.234$ (Lutz et al. 2004) and the $2-10 \mathrm{keV}$ bolometric correction $L_{\text {bol }} / L(2-10 \mathrm{keV})=20 \quad$ (e.g., Elvis et al. 1994). For the faint state, we obtained $\lambda L_{\lambda}(6 \mu \mathrm{m})=$ $(3.0 \pm 1.9) \times 10^{42} \mathrm{erg} \mathrm{s}^{-1}$, and derived $L_{\text {bol,faint }}=(1.4 \pm$ $0.9) \times 10^{43} \mathrm{erg} \mathrm{s}^{-1}$. For the bright state, we got $\lambda L_{\lambda}(6 \mu \mathrm{m})=$ $(1.0 \pm 0.1) \times 10^{44} \mathrm{erg} \mathrm{s}^{-1}$, and derived $L_{\text {bol,bright }}=(4.9 \pm$ $0.1) \times 10^{44} \mathrm{erg} \mathrm{s}^{-1}$. To estimate the peak bolometric luminosity, $L_{\text {bol,peak }}$, we fit the SED using the WISE data at the peak epoch (2017 August), combined with the DBSP spectrum and UKIRT photometry. We derived $\lambda L_{\lambda}(6 \mu \mathrm{m})=(1.6 \pm 0.2) \times$ $10^{44} \mathrm{erg} \mathrm{s}^{-1}$ at the peak epoch, thus $L_{\text {bol,peak }}=(7.5 \pm 0.1) \times$ $10^{44} \mathrm{erg} \mathrm{s}^{-1}$.

$\mathrm{J} 1657+2345$ was not detected by the ROSAT All Sky Survey observations (Boller et al. 2016) between 1990 June and 1991 August. Assuming a power-law distribution in X-ray photons with a typical photon index of $\Gamma=2$, we convert the SED-predicted

\footnotetext{
${ }^{10}$ We used the following continuum wavelength windows: 2900-3100, $3500-3700$, 3900-4260, 4430-4660, 5240-5650, 5950-6050, 6150-6250, 6800-7700, 8000-9000 А.
}

Table 4

Physical Properties of J1657+2345

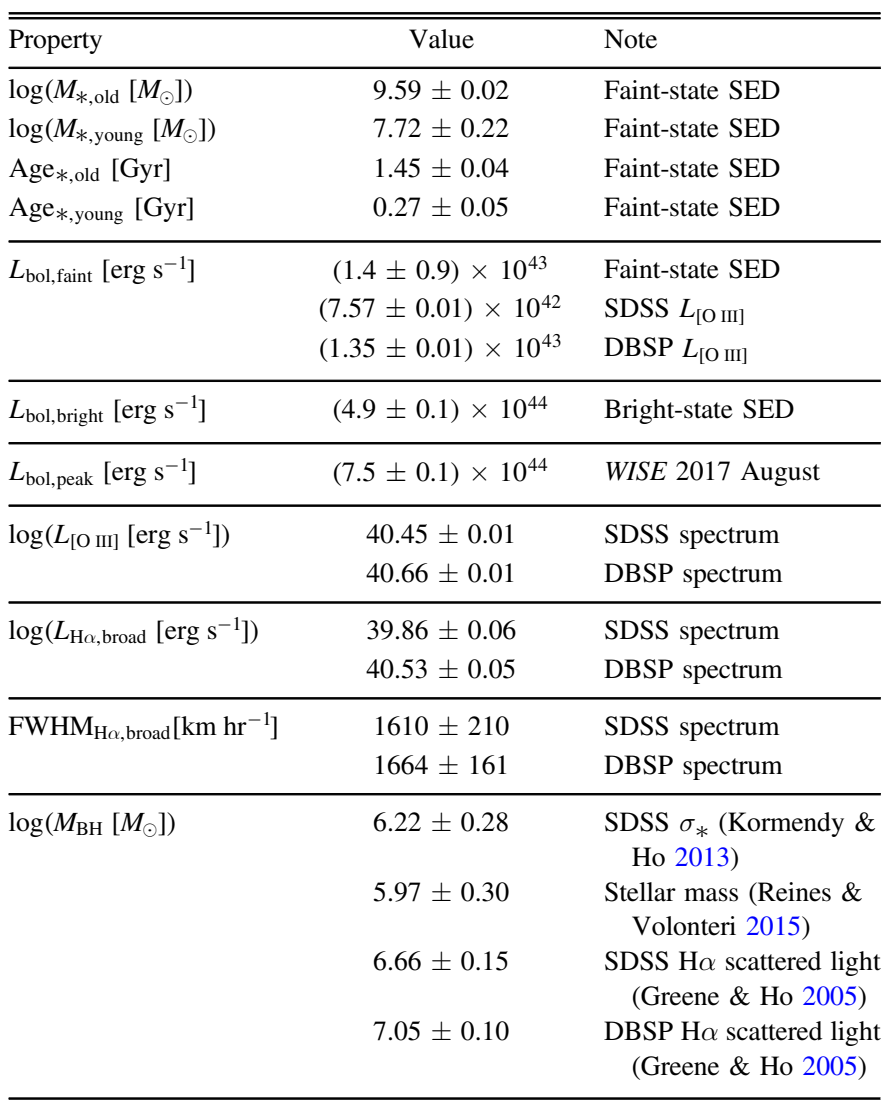

Note. All uncertainties are statistical errors only.

$L(2-10 \mathrm{keV})$ to $L(0.1-2.4 \mathrm{keV})$. We derive a flux $(0.1-2.4 \mathrm{keV})$ at the faint state of $9.1 \times 10^{-14} \mathrm{erg} \mathrm{s}^{-1} \mathrm{~cm}^{-2}$, which is below the ROSAT flux limit of a few times $10^{-13} \mathrm{erg} \mathrm{s}^{-1} \mathrm{~cm}^{-2}$.

\subsection{Spectral Properties}

To obtain emission line properties, we fit the optical spectra with stellar emission, described in Section 3.2, any residual (or scattered) power-law continuum from the AGN, ${ }^{11}$ broad-line emission, and narrow-line emission. Figure 3 shows an example of the spectral fitting to the SDSS and DBSP spectra. We summarize some spectral fitting properties in Table 4 . We only show fitting to the SDSS and DBSP spectra, because the resolution and $\mathrm{S} / \mathrm{N}$ of the spectrum taken by Xinglong $2.16 \mathrm{~m}$ telescope are lower and there is no measurable variation between this spectrum and the DBSP spectrum (see Table 2).

The $[\mathrm{O}$ III] $] \lambda 5007$ luminosity, $\left.L_{[\mathrm{O}} \mathrm{II}\right]$, from the SDSS and DBSP spectra are $10^{40.45 \pm 0.01}$ and $10^{40.66 \pm 0.01} \mathrm{erg} \mathrm{s}^{-1}$, respectively. We have scaled the $L_{[\mathrm{O} \text { III }]}$ obtained from the spectra by a factor of 1.95 to correct for aperture losses (described in Section 2.4). We estimate the bolometric luminosity from $L_{\text {[O III] }}$ following Trump et al. (2015):

$$
\frac{L_{\text {bol }}}{10^{40} \mathrm{erg} \mathrm{s}^{-1}}=112\left(\frac{L_{[\mathrm{O} \mathrm{III}]}}{10^{40} \mathrm{erg} \mathrm{s}^{-1}}\right)^{1.2} \text {. }
$$

\footnotetext{
11 However, this residual AGN continuum component is very weak and cannot be well constrained from the spectral fitting (see discussion at the end of Section 3.3).
} 


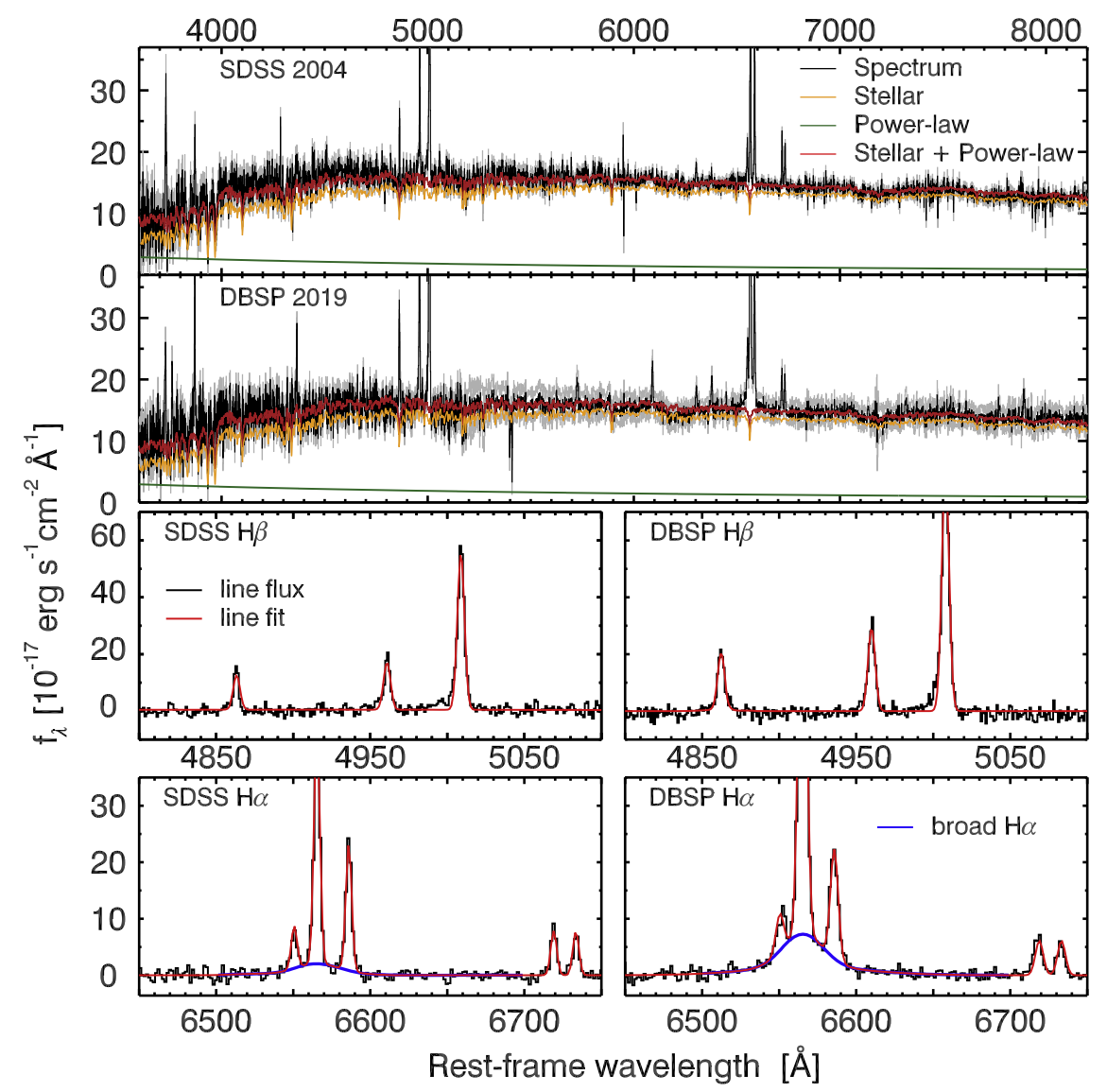

Figure 3. Spectral fits to the SDSS and DBSP spectrum. Top two panels: the data (black), uncertainties (gray), stellar (orange), power-law continuum (green), and total continuum (red). The two spectra are well fitted by the same continuum components. The power-law (AGN) contribution is weak compared to host stellar emission. Bottom four panels: zoom-in for the $\mathrm{H} \beta$ and $\mathrm{H} \alpha$ fitting to the SDSS (left) and DBSP (right) spectra. Weak broad $\mathrm{H} \alpha$ is detected in both spectra, which is consistent with scattered broad-line flux in Type 2 AGN (see text). The DBSP spectrum shows stronger broad $\mathrm{H} \alpha$ emission line (blue). No broad $\mathrm{H} \beta$ emission is detected in both spectra.

Thus the SDSS and DBSP $L_{[\mathrm{O} \text { III] }}$ corresponds to $L_{\text {bol,faint }}=$ $(7.57 \pm 0.01) \times 10^{42}$ and $(1.35 \pm 0.01) \times 10^{43} \mathrm{erg} \mathrm{s}^{-1}$, which are both consistent with that obtained from the faint-state SED in Section 3.2. The $[\mathrm{O}$ III $] \lambda 5007$ luminosity in the bright state has not yet responded to the flare, as expected from the $\sim$ kiloparsec distances of the narrow-line region gas.

There is weak broad $\mathrm{H} \alpha$ emission detected in both the SDSS and the DBSP spectra. We have tested different fitting recipes for the narrow emission lines and different extraction apertures for the DBSP spectrum and found the broad $\mathrm{H} \alpha$ measurements are robust against these details. The broad-line $\mathrm{H} \alpha$ luminosity, $L_{\mathrm{H} \alpha \text {,broad }}$, is $10^{39.86 \pm 0.06}\left(10^{40.53 \pm 0.05}\right) \mathrm{erg} \mathrm{s}^{-1}$ from the SDSS (DBSP) spectrum, and the FWHM is $1610 \pm 210(1664 \pm$ 161) $\mathrm{km} \mathrm{s}^{-1}$. On the other hand, we do not detect broad $\mathrm{H} \beta$ emission in the SDSS and DBSP spectra. The expected unobscured broad $\mathrm{H} \alpha$ luminosity, using the measurements for broad-line AGN in Shen et al. (2011), is about 1.5 dex larger than the $\left[\mathrm{O}\right.$ III] luminosity (at $L_{[\mathrm{O} \text { III }]} \sim 10^{40.5} \mathrm{erg} \mathrm{s}^{-1}$ ) in the faint state. Thus we estimate an unobscured broad $\mathrm{H} \alpha$ luminosity of $\sim 10^{42} \mathrm{erg} \mathrm{s}^{-1}$. The detected broad $\mathrm{H} \alpha$ flux in the faint state is then roughly $1 \%$ of the unobscured broad-line flux. Therefore the detected broad $\mathrm{H} \alpha$ flux is consistent with scattered light in Type 2 AGN (e.g., Zakamska et al. 2005). If this is the case, it is reasonable to see an increase in the broad $\mathrm{H} \alpha$ flux from the faint state to the bright state. The broad $\mathrm{H} \alpha$ flux of the DBSP spectrum (bright state) is a factor of $\sim 5$ of that from the SDSS spectrum (faint state). However, the MIR luminosity increased by a factor of $\sim 20$ between the faint and bright states. The UV/optical flare could last much shorter than the MIR flare (see Section 4.2). Therefore the broad $\mathrm{H} \alpha$ flux may have faded significantly when we took the DBSP spectrum.

The width and luminosity of the broad $\mathrm{H} \alpha$ line can be used to estimate the AGN black hole mass (Greene \& Ho 2005). Using the detected scattered broad $\mathrm{H} \alpha$ in the SDSS and DBSP spectra, and assuming a scattering fraction of $1 \%$ to obtain the unobscured broad $\mathrm{H} \alpha$ luminosity, we estimate a black hole mass of $10^{6.66 \pm 0.15}$ and $10^{7.05 \pm 0.10} M_{\odot}$, which are slightly higher than the black hole masses estimated from stellar mass and $\sigma_{*}$, but broadly consistent given uncertainties in the scattered light fraction and in these $\mathrm{BH}$ mass proxies.

The $M_{\mathrm{BH}}$ estimates range from $10^{5.97}$ to $10^{7.05}$ using stellar mass, velocity dispersion, and scattered broad $\mathrm{H} \alpha$ flux. We estimate the Eddington ratio $\lambda_{\mathrm{Edd}}=L_{\mathrm{bol}} / L_{\mathrm{Edd}}$, where $L_{\mathrm{Edd}}=$ $1.38 \times 10^{38}\left(M_{\mathrm{BH}} / M_{\odot}\right)$. Using the average value of $\mathrm{BH}$ mass $\left(\sim 10^{6.5} M_{\odot}\right)$ and bolometric luminosity to mitigate uncertainties in individual estimates, we estimate $\lambda_{\text {Edd }} \sim 0.02$ for the faint state, $\lambda_{\text {Edd }} \sim 1.1$ for the bright state, and $\lambda_{\text {Edd }} \sim 1.7$ for the brightest (peak WISE flux) state.

The optical continuum is dominated by stellar emission given the Type 2 AGN nature. Using a typical bolometric correction of 10 for AGN continuum luminosity at $5100 \AA, \lambda$ $L_{\lambda}(5100 \AA)$, for unobscured quasars (Shen et al. 2011), and 

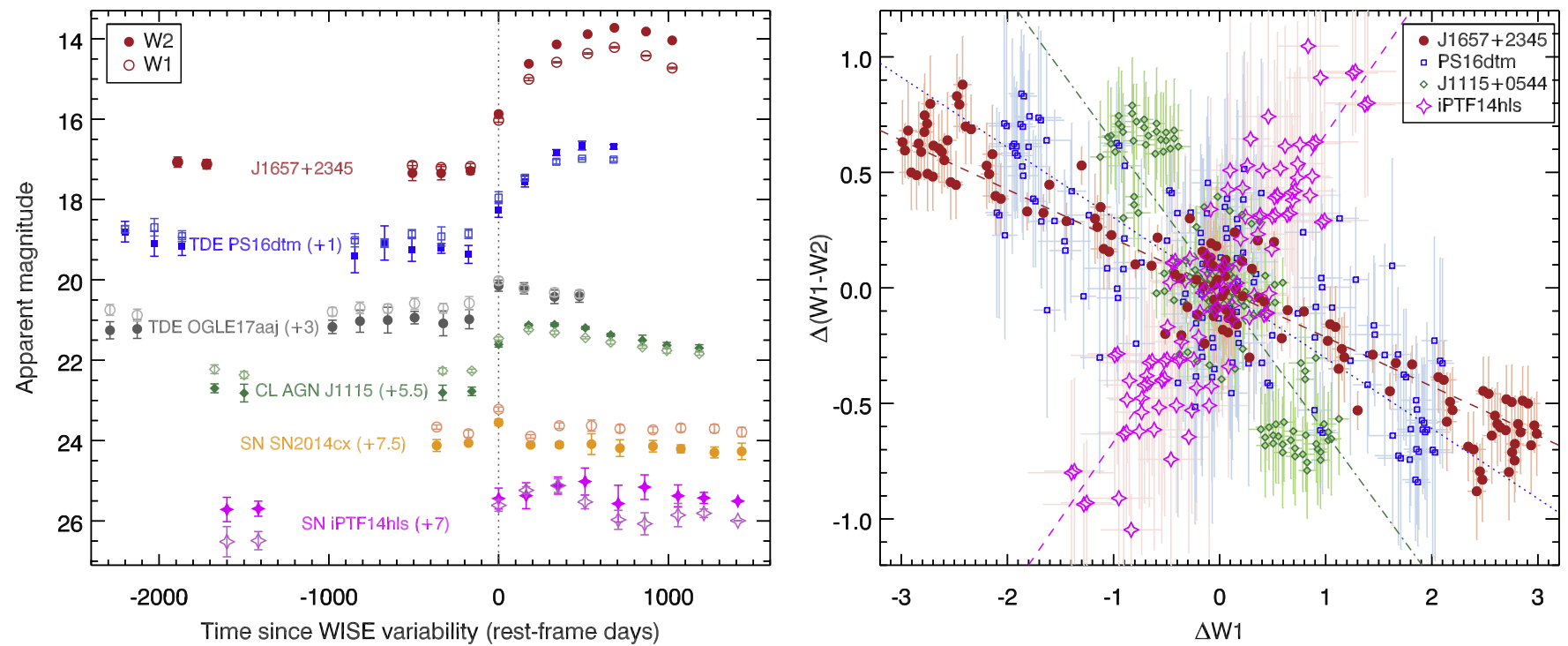

Figure 4. Left panel: MIR light curves of J1657+2345 compared with several known transients. Filled and open shapes represent $W 2$ and $W 1$ bands, respectively. From top to bottom: J1657+2345 (red), TDE PS16dtm (blue, Blanchard et al. 2017), TDE OGLE17aaj (gray, Gromadzki et al. 2019), CL AGN J1115+0544 (green, Yang et al. 2018), SN SN2014cx (orange, Guillochon et al. 2017), and SN iPTF14hls (magenta, Arcavi et al. 2017). Right panel: MIR color variability, $\Delta(W 1-W 2)$, versus magnitude variability, $\Delta W 1$, between pairs of epochs from the left panel. J1657+2345 (red filled circles), TDE PS16dtm (blue open squares), and CL AGN J1115+0544 (green open diamonds) are redder-when-brighter due to a stronger hot dust emission in bright states, while SN iPTF14hls (magenta open stars) is bluer-when-brighter, possibly as a result of no hot dust emission.

assuming the same scattering fraction of $1 \%$ as for broad $\mathrm{H} \alpha$, a bolometric luminosity $L_{\text {bol,faint }} \simeq 1.4 \times 10^{43} \mathrm{erg} \mathrm{s}^{-1}$ corresponds to a scattered AGN $\lambda L_{\lambda}(5100 \AA)$ of $1.4 \times$ $10^{40} \mathrm{erg} \mathrm{s}^{-1}$. This is less than $1 \%$ of the total observed continuum at $5100 \AA$. Thus, even if the obscured optical AGN continuum brightened by a factor of $\sim 20$, the observed total flux can only be increased by less than 0.2 mag in $r$-band, consistent with observations.

\section{Discussion}

\subsection{MIR Variability of Transients}

We compare the MIR variability of J1657+2345 with some known transient classes displaying MIR variability, including CL AGN, TDEs, and SNe. We show several examples of light curves in Figure 4. Since J1657+2345 is radio-quiet, the MIR emission is unlikely dominated by jet emission.

Yang et al. (2018) found that many CL AGN exhibit MIR variability. They are redder-when-brighter due to a stronger hot dust contribution in the $W 2$ band when the AGN activity becomes stronger.

Some studies (Dou et al. 2016, 2017; Jiang et al. 2016, 2017; van Velzen et al. 2016) reported MIR transient events following a candidate TDE flare in the optical, which were interpreted as signatures of nuclear dust reprocessing the UV/optical flare. We checked the WISE MIR light curves of additional TDEs discovered since 2014, when NEOWISE-R began continuously scanning the full sky every six months. We found that one TDE OGLE17aaj, discovered by Gromadzki et al. (2019), also displayed an MIR flare. No MIR flare was detected (at $>1 \sigma$ significance) by WISE for the other TDEs, including ASASSN14ae discovered by Holoien et al. (2014), ASASSN-15oi discovered by Holoien et al. (2016), OGLE16aaa discovered by Wyrzykowski et al. (2017), iPTF16axa discovered by Hung et al. (2017), and PS18kh discovered by Holoien et al. (2019).

We study the MIR variability of 2812 SNe discovered from 2014 January to 2018 September from an open catalog for SNe
(Guillochon et al. 2017). 58\% of them or their host galaxies were detected by WISE. $837(30 \%)$ of them were detected in more than one epoch by WISE. Among them, 115 (36) had larger than 0.5 (1) mag variability in $W 1$ band. SNe usually brighten in MIR for a shorter timescale (typically caught in one WISE epoch, i.e., less than one year) than CL AGN and TDEs (see an example of SN2014cx in the left panel of Figure 4). iPTF14hls is a peculiar SN with long-term (a few years) MIR variability after the explosion, which was classified as type II-P $\mathrm{SN}$ and interpreted as a hydrogen-rich explosion of a massive star (Arcavi et al. 2017).

However, the evolution of MIR color of SNe are generally different from those of CL AGN and TDEs. Figure 4 (right) displays the dependence of color variability $\Delta(W 1-W 2)$ on the magnitude variability $\Delta W 1$ for different populations. We fit a least-squares regression to $\Delta(W 1-W 2)$ and $\Delta W 1$ :

$$
\Delta(W 1-W 2)=A \Delta W 1 .
$$

We obtain $A=0.433 \pm 0.005$ for the $837 \mathrm{SNe}$ detected in more than one epoch by WISE, and $A=0.666 \pm 0.045$ for iPTF14hls. On the other hand, we obtain $A=-0.213 \pm 0.018$ for $\mathrm{J} 1657+2345, A=-0.304 \pm 0.022(-0.514 \pm 0.042)$ for TDE PS16dtm (OGLE16aaa), and $A=-0.629 \pm 0.064$ for CL AGN J1115+0544. CL AGN and TDEs (PS16dtm and OGLE16aaa) all display a redder-when-brighter behavior, as a consequence of stronger hot dust radiation from a nuclear dust torus that peaks at wavelengths redder than $W 2$ band. SNe, however, display a bluer-when-brighter behavior, possibly caused by the lack of hot dust contribution.

$\mathrm{J} 1657+2345$ is redder-when-brighter in MIR, with emerging strong hot dust contribution in the bright state. Thus, we speculate that the J1657+2345 MIR transient is from the dust torus of the central AGN. It is likely that the central black hole of $\mathrm{J} 1657+2345$ is increasing its accretion rate as in a CL AGN, or due to a recent TDE embedded in a highly obscuring dust torus. The flare of J1657+2345 is most likely due to TDE or 


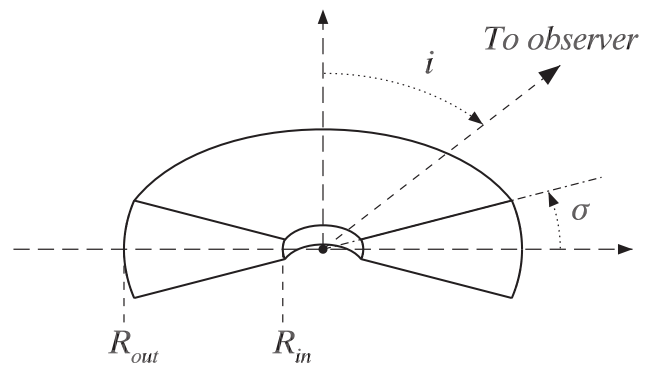

Figure 5. Simple cartoon of the dust torus geometry (e.g., Nenkova et al. 2008). The inner and outer radii are $R_{\text {in }}$ and $R_{\text {out }} \sigma$ is the half-opening angle, and $i$ is the inclination angle.

CL AGN events. It is less likely that the MIR flare of J1657 +2345 is due to a normal SN. The explosion of a massive star in the central AGN dust torus still remains a possibility based on the color variability argument. However, the detection of enhanced scattered broad $\mathrm{H} \alpha$ emission and the observed large MIR luminosity of the flare are difficult to explain with stellar explosions.

\subsection{Variability Timescales}

The MIR flare of J1657+2345 lasts more than three years. The dust torus surrounding the central SMBH responds to continuum variations and reradiates in the infrared. The continuum light arrives at different parts of the reverberating torus region at different times, and the reprocessed light reaches the observer at different later times. Therefore, the extended torus structure can lead to smoothed and stretched MIR light curves than the driving UV/optical light curve.

Here we construct a simple geometrical torus model (illustrated in Figure 5) to demonstrate the geometric effect of the dust torus on the IR echo. In our toy model, the dusty clouds are distributed between inner and outer radii $R_{\text {in }}$ and $R_{\text {out }}$, with half-opening angle $\sigma$ and inclination angle $i$. Following the methodology in Shen (2012), we describe the driving UV/ optical continuum flare as a step function at time $t=0$ followed by a constant flux increment $f_{c}$ for a period of $\Delta t$ (see Equation (2) in Shen 2012). We assume a constant density and uniform reprocessing efficiency across the entire torus region.

Figure 6 shows several examples of the responding MIR light curves for a dusty torus with $\sigma=45^{\circ}, R_{\text {in }}=2$, and $R_{\text {out }}=20$, to a step function $\mathrm{UV} /$ optical flare with a duration $\Delta t=1$. Time is in units of months and distances are in units of light-months. For such a short UV/optical flare, the resulting MIR echo can be extended to $\sim$ three years due to the spatial extension of the dust torus. The responding MIR light curves also have different shapes for different inclination angles. The average time delay between the optical/UV flare and the echoed MIR flare is determined by the radial distribution of the reprocessing material but not on inclination angle. The onset of the MIR echo is determined by $R_{\mathrm{in}}$, the torus opening angle, and the inclination angle. In our example torus model, the MIR echo should start at most at 2 months after the onset of the optical flare.

The inner radius of the torus is determined by the dust sublimation temperature as (Nenkova et al. 2008)

$$
R_{\text {in }} \simeq 0.4\left(\frac{L_{\text {bol }}}{10^{45} \mathrm{erg}^{-1}}\right)^{1 / 2}\left(\frac{1500 \mathrm{~K}}{T_{\text {sub }}}\right)^{2.6} \mathrm{pc},
$$

where $T_{\text {sub }}$ is the dust sublimation temperature and $L_{\mathrm{bol}}$ is the bolometric luminosity of the AGN. The innermost dust radius has

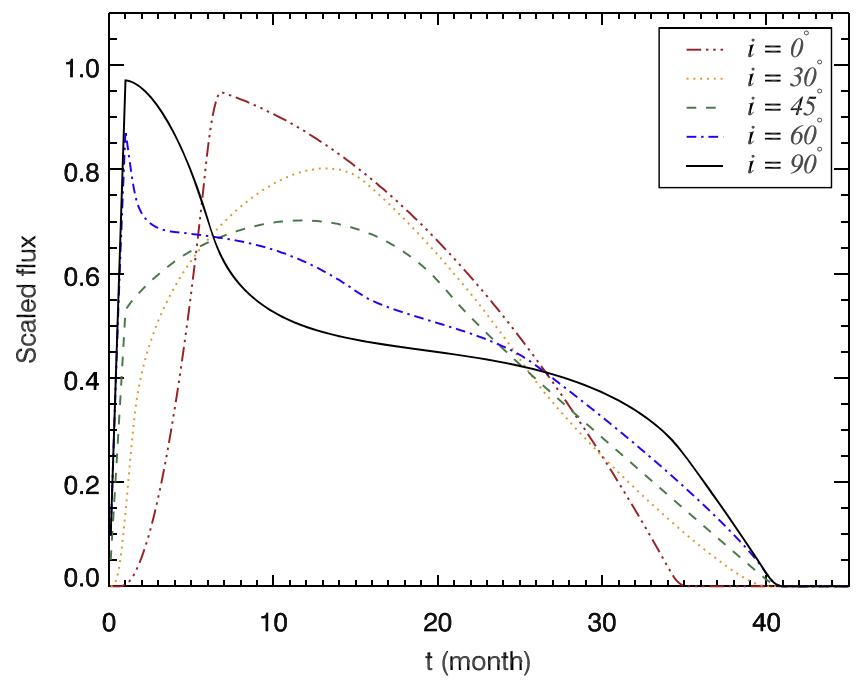

Figure 6. Torus response curves at different inclination angle $i$ of $0^{\circ}$ (red, i.e., face-on), $30^{\circ}$ (orange), $45^{\circ}$ (green), $60^{\circ}$ (blue), and $90^{\circ}$ (black, i.e., edge-on). The driving UV/optical flare is approximated by a constant flux of $f_{c}$ between $t=0$ and $t=1$ months and zero elsewhere. The responding light curves are much more extended as a consequence of the dust torus extension and the inclination angle.

now been measured by reverberation lags between optical and near-infrared ( $K$-band) for $\sim 20$ nearby AGN (e.g., Minezaki et al. 2004; Suganuma et al. 2006; Koshida et al. 2014). The inner dust radii of these AGN range from 10 to 150 light days, and correlate tightly with AGN luminosity, $L_{\mathrm{AGN}}$, as $R_{\mathrm{in}} \propto L_{\mathrm{AGN}}^{0.5}$. It is more difficult to determine the outer boundary of the dust torus. Current observations are consistent with a torus radial thickness $Y=R_{\text {out }} / R_{\text {in }}$ no more than $\sim 20-30$, and perhaps 5-10 (Nenkova et al. 2008). Thus the outer radius is a few light years.

Using the bolometric luminosity $L_{\mathrm{bol}, \text { faint }}=1.35 \times 10^{43} \mathrm{erg} \mathrm{s}^{-1}$ at the faint state, and assuming $T_{\text {sub }}=1500 \mathrm{~K}$, we obtain $R_{\text {in }} \sim 0.05 \mathrm{pc}$, corresponding to 55 lt-day. A torus radial thickness $Y=5-30$ translates to an outer boundary $R_{\text {out }}$ of approximately $0.8-4.6$ lt-yr. These estimates are similar to the values adopted for our demonstration example described earlier.

$\mathrm{J} 1657+2345$ has an estimated BH mass of $\sim 10^{6}-10^{7} M_{\odot}$ (Table 4). For a $M_{\mathrm{BH}}=10^{6} M_{\odot} \mathrm{SMBH}$, the Schwarzschild radius is $R_{S}=2 G M_{\mathrm{BH}} / c^{2}=3 \times 10^{11} \mathrm{~cm}$. The tidal disruption radius is $r_{\mathrm{T}} \simeq 5 \times 10^{12} M_{6}^{1 / 3}\left(r_{*} / r_{\odot}\right)\left(m_{*} / M_{\odot}\right)^{-1 / 3} \mathrm{~cm}$, where $M_{6}=M_{\mathrm{BH}} / 10^{6} M_{\odot}, m_{*}$ and $r_{*}$ are the mass and radius of the disrupted star (Rees 1988). Thus the tidal disruption radius of solar-type stars is much larger than the Schwarzschild radius for an $M=10^{6} M_{\odot} \mathrm{SMBH}$. The characteristic timescale of TDE, i.e., the orbital period of the most tightly bound debris, is $\Delta t=0.35 M_{7}^{1 / 2}\left(m_{*} / M_{\odot}\right)^{-1}\left(r_{*} / r_{\odot}\right)^{3 / 2} \simeq 0.1 \mathrm{yr}$, where $M_{7}=$ $M_{\mathrm{BH}} / 10^{7} M_{\odot}$ (Lodato \& Rossi 2011). Therefore, the observed multiyear MIR light curve for J1657+2345 could be the stretched response to the much shorter TDE UV/optical flare of a few months. Indeed, Mattila et al. (2018) reported a dustenshrouded TDE in a nearby merging galaxy Arp 299, whose MIR light curves are similar to that of J1657+2345. The low BH mass and the approximately Eddington-limited luminosity near the peak of the light curve makes a TDE a favorable scenario for the flare in $\mathrm{J} 1657+2345$.

Rare, rapid CL AGN phenomena have also been observed on timescales of less than $1 \mathrm{yr}$ (Gezari et al. 2017; Yang et al. 2018; Trakhtenbrot et al. 2019a; Yan et al. 2019). Thus the 
MIR flare of J1657+2345 could also be the stretched dust echo of such rapid CL AGN events. While in general CL AGN are not TDEs, some of them may be due to TDEs with observed similar decaying light curves. Indeed, the TDE scenario has been invoked to explain specific CL AGN (e.g., Merloni et al. 2015). However, the dramatic changes in the accretion flow onto the SMBH are still not fully understood (e.g., Rumbaugh et al. 2018; Dexter et al. 2019; Trakhtenbrot et al. 2019b), and it is possible that other processes can drive the required UV/ optical variability without tidally disrupting a star.

\section{Summary}

We have discovered an exceptional MIR transient in the Type 2 AGN J1657+2345, with its WISE MIR magnitudes brightened by 3 mag from 2015 to 2017 . Among more than 1.8 million galaxies in the SDSS DR14, J1657+2345 has the most extreme WISE variability amplitude. MIR variability is commonly interpreted as the dust echo of UV/optical variability. However, for this peculiar MIR transient, there is no corresponding optical photometric variability from 2003 to 2019. New optical spectra confirm that there is no significant optical variability, and new near-infrared photometry demonstrates that the MIR flare is more prominent at longer wavelengths. The lack of optical variability distinguishes $\mathrm{J} 1657+2345$ from the known population of CL AGN.

We modeled the SEDs and optical spectra of $\mathrm{J} 1657+2345$, and estimated a black mass of $\sim 10^{6}-10^{7} M_{\odot}$ and stellar mass of $\sim 10^{9.6} M_{\odot}$. The bolometric luminosity in the faint state is $1.4 \times 10^{43} \mathrm{erg} \mathrm{s}^{-1}$ and in the most recent bright state is $4.9 \times 10^{44} \mathrm{erg} \mathrm{s}^{-1}$. The peak bolometric luminosity is $7.7 \times$ $10^{44} \mathrm{erg} \mathrm{s}^{-1}$. These estimates place the accretion luminosity of the $\mathrm{BH}$ around the Eddington limit in the bright state. Based on energetic grounds and the multiyear long duration of the flare, it would be difficult to associate the MIR flare with stellar explosions and circumstellar dust reprocessing. In addition, the MIR color variability is consistent with CL AGN and TDEs, and inconsistent with normal $\mathrm{SNe}$. We also detected weak broad $\mathrm{H} \alpha$ emission in the faint and bright-state spectra, which is consistent with scattered light from the obscured broad-line region. The enhancement of the scattered broad $\mathrm{H} \alpha$ emission in the latest spectrum also favors the AGN variability and torus reprocessing scenario.

Assuming that the MIR flare comes from the AGN torus reprocessing the UV/optical variability from much closer to the $\mathrm{BH}$, we show that the extended torus geometry can reproduce the multiyear MIR light curve responding to a nuclear UV/ optical flare on much shorter timescale of a few months.

Overall our best explanation for the J1657+2345 MIR flare is that it is a rapidly turning-on AGN or TDE that is heavily obscured in the optical in a Type 2 AGN. Such objects are naturally expected as the Type 2 counterparts to the optically unobscured CL AGN population. The discovery of the J1657 +2345 flare reveals there is a population of similar nuclear flares that are missed in the optical window. A systematic search of such MIR transients among different types of galaxies will be important to understand nuclear transients in general.

We thank the referee for comments that improved the manuscript. Q.Y. and Y.S. acknowledge support from NSF grant AST-1715579. X.W., L.J., and S.Y. acknowledge support from the National Key R\&D Program of China (2016YFA0400703) and the National Science Foundation of China (11533001, 11721303, 11890693).
This publication makes use of data products from the WISE, which is a joint project of the University of California, Los Angeles, and the Jet Propulsion Laboratory/California Institute of Technology, funded by the National Aeronautics and Space Administration. This publication also makes use of data products from NEOWISE, which is a project of the Jet Propulsion Laboratory/California Institute of Technology, funded by the Planetary Science Division of the National Aeronautics and Space Administration.

Funding for the SDSS and SDSS-II has been provided by the Alfred P. Sloan Foundation, the Participating Institutions, the National Science Foundation, the U.S. Department of Energy, the National Aeronautics and Space Administration, the Japanese Monbukagakusho, the Max Planck Society, and the Higher Education Funding Council for England. The SDSS website is http://www.sdss.org/. The SDSS is managed by the Astrophysical Research Consortium for the Participating Institutions. The Participating Institutions are the American Museum of Natural History, Astrophysical Institute Potsdam, University of Basel, University of Cambridge, Case Western Reserve University, University of Chicago, Drexel University, Fermilab, the Institute for Advanced Study, the Japan Participation Group, Johns Hopkins University, the Joint Institute for Nuclear Astrophysics, the Kavli Institute for Particle Astrophysics and Cosmology, the Korean Scientist Group, the Chinese Academy of Sciences (LAMOST), Los Alamos National Laboratory, the MaxPlanck-Institute for Astronomy (MPIA), the Max-Planck-Institute for Astrophysics (MPA), New Mexico State University, Ohio State University, University of Pittsburgh, University of Portsmouth, Princeton University, the United States Naval Observatory, and the University of Washington.

The PS1 and the PS1 public science archives have been made possible through contributions by the Institute for Astronomy, the University of Hawaii, the Pan-STARRS Project Office, the Max-Planck Society and its participating institutes, the Max Planck Institute for Astronomy, Heidelberg and the Max Planck Institute for Extraterrestrial Physics, Garching, The Johns Hopkins University, Durham University, the University of Edinburgh, the Queen's University Belfast, the Harvard-Smithsonian Center for Astrophysics, the Las Cumbres Observatory Global Telescope Network Incorporated, the National Central University of Taiwan, the Space Telescope Science Institute, the National Aeronautics and Space Administration under grant No. NNX08AR22G issued through the Planetary Science Division of the NASA Science Mission Directorate, the National Science Foundation grant No. AST1238877, the University of Maryland, Eotvos Lorand University (ELTE), the Los Alamos National Laboratory, and the Gordon and Betty Moore Foundation.

The Legacy Surveys consist of three individual and complementary projects: the Dark Energy Camera Legacy Survey (DECaLS; NOAO Proposal ID 2014B-0404; PIs: David Schlegel and Arjun Dey), the Beijing-Arizona Sky Survey (BASS; NOAO Proposal ID 2015A-0801; PIs: Zhou $\mathrm{Xu}$ and Xiaohui Fan), and the Mayall z-band Legacy Survey (MzLS; NOAO Proposal ID 2016A-0453; PI: Arjun Dey). DECaLS, BASS, and MzLS together include data obtained, respectively, at the Blanco telescope, Cerro Tololo InterAmerican Observatory, National Optical Astronomy Observatory (NOAO); the Bok telescope, Steward Observatory, University of Arizona; and the Mayall telescope, Kitt Peak National Observatory, NOAO. The Legacy Surveys project is 
honored to be permitted to conduct astronomical research on Iolkam Du'ag (Kitt Peak), a mountain with particular significance to the Tohono O'odham Nation. NOAO is operated by the Association of Universities for Research in Astronomy (AURA) under a cooperative agreement with the National Science Foundation.

The CSS survey is funded by the National Aeronautics and Space Administration under grant No. NNG05GF22G issued through the Science Mission Directorate Near-Earth Objects Observations Program. The CRTS survey is supported by the U.S. National Science Foundation under grants AST-0909182.

ZTF: Based on observations obtained with the Samuel Oschin 48 inch Telescope at the Palomar Observatory as part of the Zwicky Transient Facility project. ZTF is supported by the National Science Foundation under grant No. AST-1440341 and a collaboration including Caltech, IPAC, the Weizmann Institute for Science, the Oskar Klein Center at Stockholm University, the University of Maryland, the University of Washington, Deutsches Elektronen-Synchrotron and Humboldt University, Los Alamos National Laboratories, the TANGO Consortium of Taiwan, the University of Wisconsin at Milwaukee, and Lawrence Berkeley National Laboratories. Operations are conducted by COO, IPAC, and UW.

This publication makes use of data products from the 2MASS, which is a joint project of the University of Massachusetts and the Infrared Processing and Analysis Center/California Institute of Technology, funded by the National Aeronautics and Space Administration and the National Science Foundation.

\section{ORCID iDs}

Yue Shen 주 https://orcid.org/0000-0003-1659-7035

Xin Liu (1) https://orcid.org/0000-0003-0049-5210

Xue-Bing Wu 1 (ㄴ) https://orcid.org/0000-0002-7350-6913

Linhua Jiang (i) https://orcid.org/0000-0003-4176-6486

Matthew J. Graham (1) https://orcid.org/0000-0002-3168-0139

Su Yao (1) https://orcid.org/0000-0002-9728-1552

\section{References}

Abazajian, K. N., Adelman-McCarthy, J. K., Agüeros, M. A., et al. 2009, ApJS, 182, 543

Abolfathi, B., Aguado, D. S., Aguilar, G., et al. 2018, ApJS, 235, 42

Arcavi, I., Gal-Yam, A., Sullivan, M., et al. 2014, ApJ, 793, 38

Arcavi, I., Howell, D. A., Kasen, D., et al. 2017, Natur, 551, 210

Assef, R. J., Prieto, J. L., Stern, D., et al. 2018, ApJ, 866, 26

Bellm, E. C., Kulkarni, S. R., Graham, M. J., et al. 2019, PASP, 131, 018002

Bessiere, P. S., Tadhunter, C. N., Ramos Almeida, C., Villar Martín, M., \& Cabrera-Lavers, A. 2017, MNRAS, 466, 3887

Blanchard, P. K., Nicholl, M., Berger, E., et al. 2017, ApJ, 843, 106 Boller, T., Freyberg, M. J., Trümper, J., et al. 2016, A\&A, 588, A103

Bruzual, G., \& Charlot, S. 2003, MNRAS, 344, 1000

Chabrier, G. 2003, PASP, 115, 763

Chambers, K. C., Magnier, E. A., Metcalfe, N., et al. 2016, arXiv:1612.05560

Cid Fernandes, R., Stasińska, G., Schlickmann, M. S., et al. 2010, MNRAS, 403, 1036

Dexter, J., Xin, S., Shen, Y., et al. 2019, arXiv:1906.10138

Dey, A., Schlegel, D. J., Lang, D., et al. 2019, AJ, 157, 168

Dou, L., Wang, T., Yan, L., et al. 2017, ApJL, 841, L8

Dou, L., Wang, T.-g., Jiang, N., et al. 2016, ApJ, 832, 188

Drake, A. J., Djorgovski, S. G., Mahabal, A., et al. 2009, ApJ, 696, 870

Dwek, E., Dinerstein, H. L., Gillett, F. C., Hauser, M. G., \& Rice, W. L. 1987, ApJ, 315, 571

Elvis, M., Wilkes, B. J., McDowell, J. C., et al. 1994, ApJS, 95, 1

Fan, Z., Wang, H., Jiang, X., et al. 2016, PASP, 128, 115005

Filippenko, A. V. 1997, ARA\&A, 35, 309
Fukugita, M., Ichikawa, T., Gunn, J. E., et al. 1996, AJ, 111, 1748

Gezari, S., Chornock, R., Rest, A., et al. 2012, Natur, 485, 217

Gezari, S., Hung, T., Cenko, S. B., et al. 2017, ApJ, 835, 144

Greene, J. E., \& Ho, L. C. 2005, ApJ, 630, 122

Gromadzki, M., Hamanowicz, A., Wyrzykowski, L., et al. 2019, A\&A, 622, L2

Guillochon, J., Parrent, J., Kelley, L. Z., \& Margutti, R. 2017, ApJ, 835, 64

Heger, A., Fryer, C. L., Woosley, S. E., Langer, N., \& Hartmann, D. H. 2003, ApJ, 591, 288

Hewett, P. C., Warren, S. J., Leggett, S. K., \& Hodgkin, S. T. 2006, MNRAS, 367,454

Hillebrandt, W., \& Niemeyer, J. C. 2000, ARA\&A, 38, 191

Holoien, T. W. S., Huber, M. E., Shappee, B. J., et al. 2019, ApJ, 880, 120

Holoien, T. W. S., Kochanek, C. S., Prieto, J. L., et al. 2016, MNRAS, 463,3813

Holoien, T. W. S., Prieto, J. L., Bersier, D., et al. 2014, MNRAS, 445, 3263

Hönig, S. F., \& Kishimoto, M. 2017, ApJL, 838, L20

Hung, T., Gezari, S., Blagorodnova, N., et al. 2017, ApJ, 842, 29

Jiang, N., Dou, L., Wang, T., et al. 2016, ApJL, 828, L14

Jiang, N., Wang, T., Yan, L., et al. 2017, ApJ, 850, 63

Kauffmann, G., Heckman, T. M., Tremonti, C., et al. 2003, MNRAS, 346,1055

Komossa, S. 2015, JHEAp, 7, 148

Komossa, S., Zhou, H., Wang, T., et al. 2008, ApJL, 678, L13

Kormendy, J., \& Ho, L. C. 2013, ARA\&A, 51, 511

Koshida, S., Minezaki, T., Yoshii, Y., et al. 2014, ApJ, 788, 159

LaMassa, S. M., Cales, S., Moran, E. C., et al. 2015, ApJ, 800, 144

Lodato, G., \& Rossi, E. M. 2011, MNRAS, 410, 359

Lutz, D., Maiolino, R., Spoon, H. W. W., \& Moorwood, A. F. M. 2004, A\&A, 418, 465

MacLeod, C. L., Green, P. J., Anderson, S. F., et al. 2019, ApJ, 874, 8

MacLeod, C. L., Ross, N. P., Lawrence, A., et al. 2016, MNRAS, 457, 389

Mainzer, A., Bauer, J., Cutri, R. M., et al. 2014, ApJ, 792, 30

Mainzer, A., Grav, T., Bauer, J., et al. 2011, ApJ, 743, 156

Mattila, S., Pérez-Torres, M., Efstathiou, A., et al. 2018, Sci, 361, 482

Merloni, A., Dwelly, T., Salvato, M., et al. 2015, MNRAS, 452, 69

Minezaki, T., Yoshii, Y., Kobayashi, Y., et al. 2004, ApJL, 600, L35

Nenkova, M., Sirocky, M. M., Nikutta, R., Ivezić, Ž, \& Elitzur, M. 2008, ApJ, 685,160

Oke, J. B., \& Gunn, J. E. 1983, ApJ, 266, 713

Rees, M. J. 1988, Natur, 333, 523

Reines, A. E., \& Volonteri, M. 2015, ApJ, 813, 82

Ross, N. P., Ford, K. E. S., Graham, M., et al. 2018, MNRAS, 480, 4468

Rumbaugh, N., Shen, Y., Morganson, E., et al. 2018, ApJ, 854, 160

Runnoe, J. C., Cales, S., Ruan, J. J., et al. 2016, MNRAS, 455, 1691

Shangguan, J., \& Ho, L. C. 2019, ApJ, 873, 90

Shangguan, J., Ho, L. C., \& Xie, Y. 2018, ApJ, 854, 158

Shen, Y. 2012, ApJ, 757, 152

Shen, Y., Richards, G. T., Strauss, M. A., et al. 2011, ApJS, 194, 45

Sheng, Z., Wang, T., Jiang, N., et al. 2017, ApJL, 846, L7

Skrutskie, M. F., Cutri, R. M., Stiening, R., et al. 2006, AJ, 131, 1163

Stern, D., McKernan, B., Graham, M. J., et al. 2018, ApJ, 864, 27

Suganuma, M., Yoshii, Y., Kobayashi, Y., et al. 2006, ApJ, 639, 46

Thomas, D., Steele, O., Maraston, C., et al. 2013, MNRAS, 431, 1383

Tody, D. 1986, Proc. SPIE, 627, 733

Tody, D. 1993, in ASP Conf. Ser. 52, Astronomical Data Analysis Software and Systems II, ed. R. J. Hanisch, R. J. V. Brissenden, \& J. Barnes (San Francisco, CA: ASP), 173

Trakhtenbrot, B., Arcavi, I., MacLeod, C. L., et al. 2019a, ApJ, 883, 94

Trakhtenbrot, B., Arcavi, I., Ricci, C., et al. 2019b, NatAs, 3, 242

Trump, J. R., Sun, M., Zeimann, G. R., et al. 2015, ApJ, 811, 26

Urry, C. M., \& Padovani, P. 1995, PASP, 107, 803

van Velzen, S., Farrar, G. R., Gezari, S., et al. 2011, ApJ, 741, 73

van Velzen, S., Mendez, A. J., Krolik, J. H., \& Gorjian, V. 2016, ApJ, 829, 19

Wang, T., Yan, L., Dou, L., et al. 2018, MNRAS, 477, 2943

Wang, T.-G., Zhou, H.-Y., Komossa, S., et al. 2012, ApJ, 749, 115

Wright, E. L., Eisenhardt, P. R. M., Mainzer, A. K., et al. 2010, AJ, 140, 1868

Yan, L., Wang, T., Jiang, N., et al. 2019, ApJ, 874, 44

Yang, Q., Wu, X.-B., Fan, X., et al. 2018, ApJ, 862, 109

York, D. G., Adelman, J., Anderson, J. E., Jr., et al. 2000, AJ, 120, 1579

Zakamska, N. L., Schmidt, G. D., Smith, P. S., et al. 2005, AJ, 129, 1212

Zhao, D., Ho, L. C., Zhao, Y., Shangguan, J., \& Kim, M. 2019, ApJ, 877, 52

Zhuang, M.-Y., Ho, L. C., \& Shangguan, J. 2018, ApJ, 862, 118

Wyrzykowski, Ł., Zieliński, M., Kostrzewa-Rutkowska, Z., et al. 2017, MNRAS, 465, L114 